\title{
Wind tunnel measurements of turbulent boundary layer flows over arrays of ribs and cubes
}

\author{
Ziwei Mo and Chun-Ho Liu* (i)
}

\begin{abstract}
Understanding the effect of building morphology on the flow aloft is important to the ventilation and pollutant removal in cities. This study examines the dynamics over hypothetical urban areas in isothermal conditions using wind tunnel experiments. Different configurations of rib-type and cube-type arrays are designed to model hypothetical rough urban surfaces. The mean and fluctuating velocities are measured by hot-wire anemometry with $X$-wire probes. The results show that significant variations of fluctuating velocities and momentum fluxes are clearly observed in the near-wall region, depicting the inhomogeneous flow in response to the presence of roughness elements in the lower part of turbulent boundary layer. Comparing the variables over different rough surfaces, the roof-level fluctuating velocities and momentum fluxes increase with increasing surface roughness. Quadrant analyses and frequency spectra collectively suggest that the fresh air entrainment and aged air removal are enhanced over rougher surfaces. Larger energy-carrying turbulence motions contribute mostly to the more efficient ventilation over urban areas.
\end{abstract}

Keywords: Frequency spectra, Momentum fluxes, Quadrant analyses, Turbulent flows, Wind tunnel laboratory experiments

\section{Background}

In the presence of building obstacles in urban areas, the atmospheric boundary layer (ABL) is developed similar to the rough-wall turbulent boundary layer (TBL; Pope 2000). The flow structure and turbulence behavior are highly modified over different types of surface roughness (Jiménez 2004). It is, therefore, important to study the flow characteristics in the TBLs over rough surfaces.

Wind tunnel experiments are commonly performed to examine the turbulent flows over rough surfaces (Raupach et al. 1991). Scaling down the dimensions of realistic urban areas in a wind tunnel offers a cost-effective platform for sensitivity tests with full control of variables and boundary conditions (Cermak 1981). A series of wind tunnel studies have been carried out to demystify

\footnotetext{
${ }^{*}$ Correspondence: liuchunho@graduate.hku.hk

Department of Mechanical Engineering, The University of Hong Kong,
}

7/F, Haking Wong Building, Pokfulam Road, Hong Kong, China the effects of roughness-element configurations on the flows in rough-wall TBLs (Britter and Hanna 2003; Salizzoni et al. 2008; Liu et al. 2015). ABL velocity profiles are examined over arrays of ribs (Salizzoni et al. 2008; Ho and Liu 2017) and arrays of cubes (Cheng and Castro 2002a, b). Some of the aerodynamic parameters, such as displacement height $d$ and roughness length $z_{0}$, were contrasted over different surface configurations. The effect of roughness elements on the roughness sublayer (RSL) was also investigated (Placidi and Ganapathisubramani 2015). Besides, turbulence structure was characterized by autocorrelation, quadrant analyses as well as spectra over cube-type arrays (Castro et al. 2006). These experimental studies have enriched our understanding of turbulent flows over rough-wall TBLs. However, more wind tunnel results are needed to study the effect of surface configurations on the turbulence behavior and the associated street-level ventilation over urban areas. 
In this study, a series of wind tunnel experiments are carried out to examine the flows in the TBLs over ribtype and cube-type arrays. Square aluminum bars and LEGO $^{\mathrm{TM}}$ bricks are used to fabricate different configurations of hypothetical urban areas. The profiles of mean wind speeds and turbulence are sampled in each repeating unit of roughness element. The effect of sampling position and rough-surface configurations on the flows is contrasted. Quadrant analyses and frequency spectra are performed as well to elucidate the scale of motions governing the roof-level ventilation mechanism over urban areas.

\section{Methods}

An open-circuit, isothermal wind tunnel, which is located in the Department of Mechanical Engineering, The University of Hong Kong (Ho and Liu 2017; Mo and Liu 2018), is employed to perform the laboratory-scale experiments (Fig. 1a). The dimensions of its test section are $6 \mathrm{~m}$ long, $0.56 \mathrm{~m}$ wide and $0.56 \mathrm{~m}$ high. Repeating units of the reduced-scale models are glued on the whole floor to generate a fully developed TBL (Kozmar 2010). The free-stream wind speed $U_{\infty}$ in the wind tunnel is being monitored by a pitot-static tube installed upstream of the test section thorough a set of experiments to maintain steady wind conditions. The wind tunnel is equipped with a digital traversed system operated by National Instruments (NI 2018) motion control modules (PCI-7390) for sensor positioning whose spatial resolution is $1 \mathrm{~mm}$ in both streamwise $x$ and vertical $z$ direction.

\section{Roughness elements}

Models of hypothetical urban areas are fabricated by idealized roughness elements in the wind tunnel test section. Two types of rough surfaces are considered in this study, namely, rib-type arrays and cube-type arrays. The rib-type arrays are assembled by square aluminum bars of size $l(=560 \mathrm{~mm}$; long $) \times h(=9 \mathrm{~mm}$; wide $) \times h$ (=9 mm; high). The ribs are placed evenly apart in crossflows, spanning the full width of the wind tunnel test section. Ten configurations of rib-type arrays are adopted by adjusting the separation between the ribs $w$. The roughness-element-height-to-separation (aspect) ratios (AR) are equal to $1,1 / 2,1 / 3,1 / 4,1 / 5,1 / 6,1 / 8,1 / 10,1 / 12$, and $1 / 15$. For the cube-type arrays, roughness elements are assembled by staggering LEGO $^{\circledR}$ bricks on a LEGO ${ }^{\circledR}$ baseboard. The size of each piece of $\mathrm{LEGO}^{\circledR}$ brick is $l$ $(=16 \mathrm{~mm}$; long $) \times l(=16 \mathrm{~mm}$; wide $) \times h(=11.4 \mathrm{~mm}$; high, including the studs at the top). The separation among the $\mathrm{LEGO}^{\circledR}$ bricks is varied in the streamwise $x$ direction, covering $h: l, h: 2 l, h: 3 l, h: 4 l, h: 5 l, h: 6 l, h: 7 l$ and $h: 9 l$. In addition, the height of cube-type arrays is increased by mounting double $(h: 4 l-D)$, triple $(h: 4 l-T)$ and quadruple $(h: 4 l-Q)$ layers of $\mathrm{LEGO}^{\circledR}$ bricks on the $h: 4 l$ configuration. Examples of the roughness configurations $(\mathrm{AR}=1 / 2, \mathrm{AR}=1 / 4, h: 2 l$, and $h: 6 l)$ are shown in

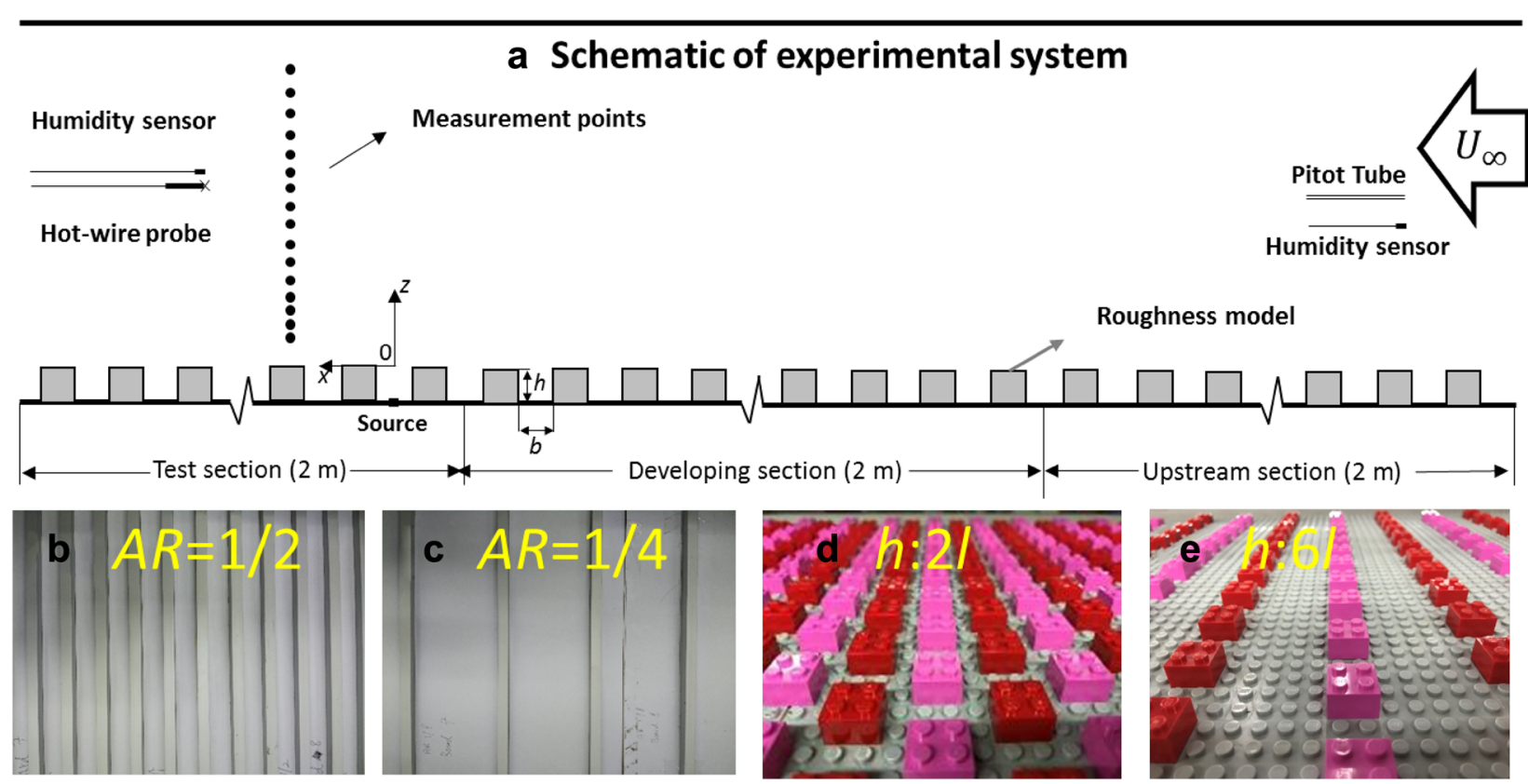

Fig. 1 Schematic of wind tunnel (a) and configuration of roughness element. $\mathbf{b} A R=1 / 2, \mathbf{c} A R=1 / 3, \mathbf{d} h: 2 /$ and $\mathbf{e} h: 6 /$ 
Fig. 1b-e. A total of eleven configurations of cube-type array of roughness element are employed in the wind tunnel measurements.

\section{Velocity measurements}

The mean and fluctuating velocities are measured by a constant-temperature hot-wire anemometer (CTA). A $X$-wire probe is mounted to measure streamwise $u$ and vertical $w$ velocity components. The sensing elements are made of 5- $\mu \mathrm{m}$ (diameter) platinum-plated tungsten wires with 2-mm active length by copper electroplating. The included angle between the two wires is $100^{\circ}$ $\left(>90^{\circ}\right)$ that helps reduce the error due to inadequate yaw response in elevated turbulence intensity in near-wall region (Krogstad et al. 1992; Perry et al. 1987; Cheng and Castro 2002a, b). The CTA analog signal is digitized by a 24-bit NI data acquisition module (NI 9239) mounted in a NI CompactDAQ chassis (NI cDAQ-9188). The digital data are then collected by LabVIEW software on a digital computer. The (voltage) signal is then converted to velocity based on the universal calibration scheme (Bruun 1971). The CTA-measured velocity is compared with the velocity measured by the (upstream) pitot-static tube in which the regression coefficient $R^{2}$ is up to 0.999. Seven vertical profiles are collected for each repeating unit of roughness element (Fig. 2), covering the top of roughness elements (P1 and P7), cavity top (P3, P4, and P5), leeward edge (P2), and windward edge (P6). A total of 96 sampling points are probed in each vertical profile, ranging from the roughness element height $z=h$ to the wall-normal distance over the TBLs $z=350 \mathrm{~mm}$. The sampling time is $66 \mathrm{~s}$ at each point and the sampling frequency is $2000 \mathrm{~Hz}$. Over $2^{17}$ data are collected at each point and the sampling duration for each case of array configuration is over $12 \mathrm{~h}$.

\section{Results and discussion}

Dynamics over different rough surfaces are analyzed based on the wind tunnel measurements. In the following section, overbar $\bar{\bullet}$, angle bracket $\langle\bullet\rangle$ and double prime $\bullet$ $(=\bullet-\langle\bar{\bullet}\rangle)$ denote the temporal average, spatial average and fluctuating component, respectively. Temporal average $\boldsymbol{} \boldsymbol{}$ is the averaged property during the sampling duration at each point while spatial average $\langle\bullet\rangle$ is the averaged property at wall-normal distance $z$ of seven vertical profiles measured at different streamwise positions $x$.

\section{Turbulent boundary layer parameters}

Based on the velocity measurements, the TBL parameters in this study are tabulated in Table 1. The TBL thickness $\delta$ is defined by at the wall-normal distance $z$ where the spatio-temporal average of mean wind speeds converge to $99 \%$ of the free-stream one

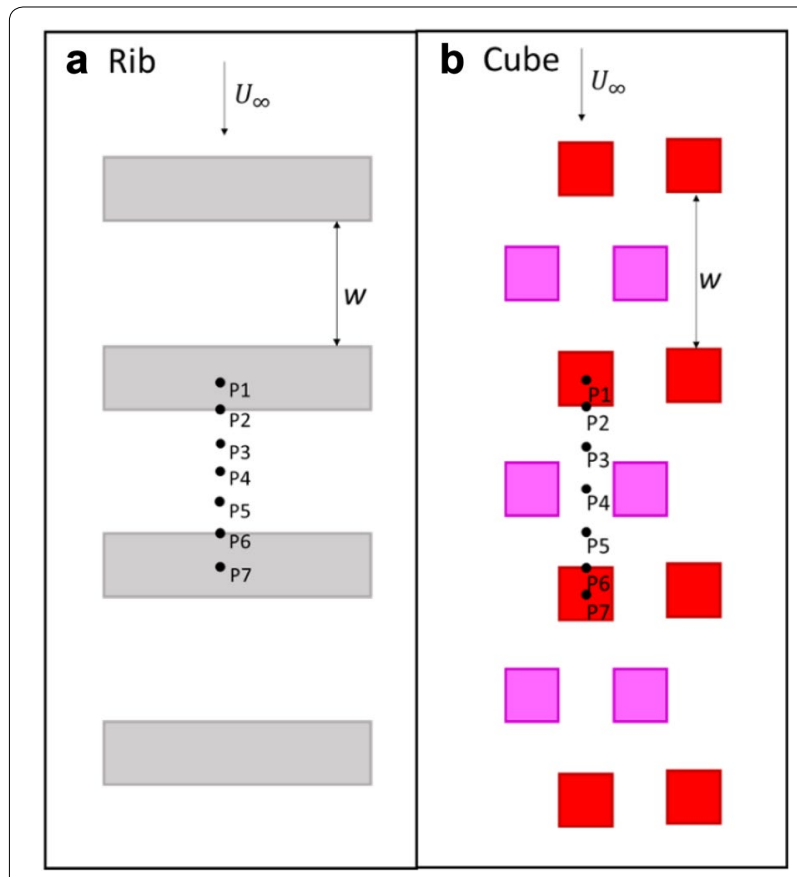

Fig. 2 Plan view of a rib-type and $\mathbf{b}$ cube-type arrays. Also shown are the sampling positions of the vertical profiles over rough surfaces (black solid circles)

$\left.\langle\bar{u}\rangle\right|_{z=\delta}=0.99 U_{\infty}$ (Cheng and Castro 2002a, b). In this study, the free-stream wind speeds at the TBL top are in the ranges of $8-9 \mathrm{~m} \mathrm{~s}^{-1}$ and $10-11 \mathrm{~m} \mathrm{~s}^{-1}$, respectively, for the rib-type and cube-type arrays. The TBL thickness over rib-type arrays is in the range of $219 \mathrm{~mm}$ $(12 h) \leq \delta \leq 304 \mathrm{~mm}(16 h)$ that is larger than its cubetype counterpart which is in the range of $135 \mathrm{~mm}$ $(5 h) \leq \delta \leq 219 \mathrm{~mm}(14 h)$. The thicker TBLs over ribtype arrays are caused by the higher obstacle height together with the elevated aerodynamic resistance. The Reynolds number based on free-stream wind speed and TBL thickness $\operatorname{Re}_{\infty}\left(=U_{\infty} \delta / v\right)$ is in the range of $125,000 \leq \operatorname{Re}_{\infty} \leq 277,000$ for rib-type arrays and $135,000 \leq \operatorname{Re}_{\infty} \leq 255,000$ for cube-type arrays that is sufficiently high to neglect the effect of molecular viscosity in the analyses.

The friction velocity is defined as $u_{*}=\left(\tau_{w} / \rho\right)^{1 / 2}$ where $\tau_{w}$ is the total shear stress on the rough surface and $\rho$ the fluid density. In the wind tunnel measurements, the friction velocity is commonly estimated by the relationship $u_{*}\left(=\left\langle\overline{u^{\prime \prime} w^{\prime \prime}}\right\rangle^{1 / 2}\right)$ by averaging the turbulent momentum flux over the entire rough surface (Cheng and Castro 2002a; Salizzoni et al. 2008). Cheng et al. (2007) reported that $u_{*}$ was underestimated by $25 \%$ over staggered arrays of cubical elements based on averaging $\overline{u^{\prime \prime} w^{\prime \prime}}$ in the inertia sublayer (ISL) compared with that of direct drag measurement. In addition, $u_{*}$ is 
Table 1 Parameters in the turbulent boundary layers over different rough surfaces

\begin{tabular}{|c|c|c|c|c|c|c|c|c|}
\hline Rough surfaces & $h\left(\times 10^{-3} \mathrm{~m}\right)$ & $w\left(\times 10^{-3} \mathrm{~m}\right)$ & $\delta\left(\times 10^{-3} \mathrm{~m}\right)$ & $U_{\infty}\left(\mathrm{m} \mathrm{s}^{-1}\right)$ & $u_{*}\left(\mathrm{~m} \mathrm{~s}^{-1}\right)$ & $C_{d}\left(\times 10^{-3}\right)$ & $d\left(\times 10^{-3} \mathrm{~m}\right)$ & $z_{0}\left(\times 10^{-3} \mathrm{~m}\right)$ \\
\hline \multicolumn{9}{|l|}{ Rib-type arrays } \\
\hline$A R=1 / 1$ & 19 & 19 & 219 & 8.0 & 0.36 & 4.1 & 6.0 & 0.04 \\
\hline$A R=1 / 2$ & 19 & 38 & 244 & 8.0 & 0.45 & 6.5 & 6.9 & 0.44 \\
\hline$A R=1 / 3$ & 19 & 57 & 248 & 8.4 & 0.52 & 7.6 & 5.9 & 0.63 \\
\hline$A R=1 / 4$ & 19 & 76 & 283 & 8.5 & 0.56 & 8.7 & 7.0 & 0.81 \\
\hline$A R=1 / 5$ & 19 & 95 & 284 & 8.5 & 0.59 & 9.6 & 6.2 & 1.04 \\
\hline$A R=1 / 6$ & 19 & 114 & 294 & 8.5 & 0.60 & 9.9 & 6.1 & 0.98 \\
\hline$A R=1 / 8$ & 19 & 152 & 294 & 8.4 & 0.60 & 10.1 & 4.0 & 1.04 \\
\hline$A R=1 / 10$ & 19 & 190 & 304 & 9.1 & 0.65 & 10.1 & 8.3 & 0.80 \\
\hline$A R=1 / 12$ & 19 & 228 & 304 & 9.1 & 0.67 & 10.8 & 13.6 & 0.85 \\
\hline$A R=1 / 15$ & 19 & 285 & 293 & 9.0 & 0.64 & 10.1 & 11.6 & 0.73 \\
\hline \multicolumn{9}{|l|}{ Cube-type arrays } \\
\hline$h: l$ & 11.4 & 16 & 135 & 10.0 & 0.42 & 3.6 & 5.2 & 0.02 \\
\hline$h: 21$ & 11.4 & 32 & 165 & 10.9 & 0.53 & 4.8 & 5.8 & 0.08 \\
\hline$h: 31$ & 11.4 & 48 & 165 & 10.8 & 0.54 & 4.9 & 5.3 & 0.09 \\
\hline$h: 4 l$ & 11.4 & 64 & 165 & 10.8 & 0.56 & 5.5 & 5.6 & 0.13 \\
\hline$h: 5 /$ & 11.4 & 80 & 160 & 10.6 & 0.54 & 5.2 & 5.4 & 0.11 \\
\hline$h: 6 /$ & 11.4 & 96 & 165 & 10.6 & 0.54 & 5.1 & 5.3 & 0.10 \\
\hline$h: 71$ & 11.4 & 112 & 160 & 10.6 & 0.53 & 5.0 & 5.0 & 0.09 \\
\hline$h: 9 /$ & 11.4 & 144 & 155 & 10.7 & 0.51 & 4.5 & 4.8 & 0.06 \\
\hline$h: 4 l-D$ & 21 & 64 & 190 & 10.8 & 0.60 & 6.3 & 5.8 & 0.23 \\
\hline$h: 4 l-T$ & 30.6 & 64 & 215 & 11.1 & 0.66 & 7.1 & 5.1 & 0.37 \\
\hline$h: 4 l-Q$ & 40.2 & 64 & 219 & 11.2 & 0.70 & 7.9 & 3.6 & 0.52 \\
\hline
\end{tabular}

obtained by assuming it to be the maximum of Reynolds shear stresses in the same studies, and comparable with a corrected estimate value defined as $(1+0.25) \times\left.\left\langle\overline{u^{\prime \prime} w^{\prime \prime}}\right\rangle^{1 / 2}\right|_{\text {ISL }}$ (Manes et al. 2011; Placidi and Ganapathisubramani 2015; Cheng et al. 2007). In this study, we adopt the conventional method by assuming that $u_{*}$ is equal to the peaked $\left\langle\overline{u^{\prime \prime} w^{\prime \prime}}\right\rangle^{1 / 2}$. Although this would introduce error (within 25\% uncertainty) in estimating the value of $u$, the variation pattern of $u_{*}$ in this study will not be significantly affected as a consistent method is used among the testing cases. The friction velocity $u$ over rib-type and cube-type arrays is estimated in the ranges of $0.36-$ $0.67 \mathrm{~m} \mathrm{~s}^{-1}$ and $0.42-0.70 \mathrm{~m} \mathrm{~s}^{-1}$, respectively (Table 1 ). Using $u_{*}$ as the slope, the other two key rough-TBL parameters, roughness length $z_{0}$ and displacement height $d$, are determined by the best fit of the wind-tunnel-measured mean wind speed profiles to the theoretical logarithmic law of the wall (log law). As shown in Table 1 , the displacement height is in the range of $4.1 \mathrm{~mm}(0.2 h) \leq d \leq 13.6 \mathrm{~mm}(0.72 h)$ over the rib-type arrays and $3.6 \mathrm{~mm}(0.09 h) \leq d \leq 5.8 \mathrm{~mm}(0.5 h)$ over the cube-type arrays. The roughness length $z_{0}$ is much smaller, ranging from $0.04 \mathrm{~mm}(0.002 h)$ to $1.04 \mathrm{~mm}$ (0.06h) over rib-type arrays and from $0.02 \mathrm{~mm}(0.002 h)$ to $0.52 \mathrm{~mm}(0.013 h)$ over cube-type arrays. Drag coefficient $C_{d}\left(=2 u_{*}^{2} / U_{\infty}^{2}\right)$ is commonly used to measure the aerodynamic resistance for flows over (non-smooth) solid boundaries. It is found to be $4.1 \times 10^{-3}$ $\leq C_{d} \leq 10.1 \times 10^{-3}$ over rib-type arrays and $3.6 \times 10^{-3} \leq C_{d} \leq 7.9 \times 10^{-3}$ over cube-type arrays.

The variations of TBL parameters, such as $\delta, z_{0}$, and $d$, are closely influenced by the configurations of surface roughness. The relationship between TBL parameters and the aspect ratio (rib-type arrays) or packing density (frontal and plan solidities of cube-type arrays) has been evaluated in previous studies (Cheng et al. 2007; Placidi and Ganapathisubramani 2015; Ho and Liu 2017). In this paper, we use the drag coefficient $C_{d}$ as the quantitative indicator of different configurations of rough surface. Figure 3 plots $z_{0}, d$, and $\delta$ against $C_{d}$ in both dimensional and dimensionless form. The data over ribs and cubes obtained from previous studies (Cheng and Castro 2002a; Salizzoni et al. 2008; Placidi and Ganapathisubramani 2015) are also compared. There is a noticeable trend that the $z_{0}$ increases with increasing $C_{d}$ (Fig. 3a). However, the increasing rate of $z_{0} / h$ for cube-type elements and ribtype elements becomes significantly different. It is thus suggested that the roughness-element height $h$ is not the 

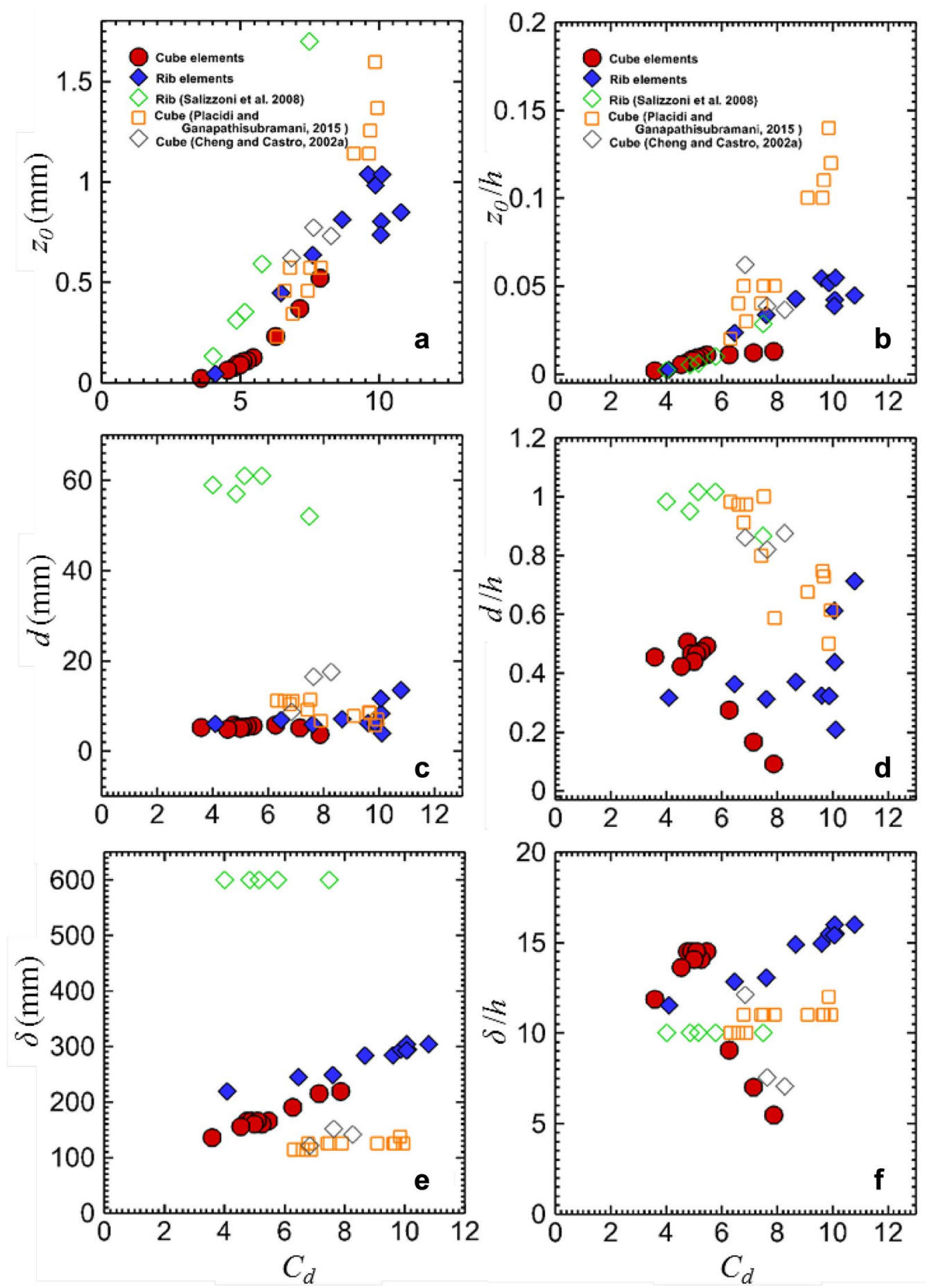

Fig. 3 Comparison of a roughness length $z_{0}, \mathbf{c}$ displacement height $d$ and $\mathbf{d}$ boundary layer thickness $\delta$ plotted against drag coefficient $C_{d}$. Also shown in (b, $\mathbf{d}$ and $\mathbf{f})$ are the corresponding properties normalized by the roughness-element height $h$

most appropriate characteristic length scale for normalization (Fig. 3b). Displacement height, $d$, does not show any obvious increase with increasing $C_{d}$ for both rib-type and cube-type elements while its dimensionless form $d / h$ varies significantly (Fig. 3c, d). It is in turn suggested that $d$ has insignificant relation with $C_{d}$. It should be noted that there is large uncertainty in the estimate of $d$ by the best fit of measured mean wind profile to the log law. 
The TBL thickness $\delta$ increases slightly with increasing $C_{d}$ that suggests a possible relation between them (Fig. 3e). However, scatters of $\delta$ scaled by the roughness element height $h$ are found with increasing $C_{d}$ for the cube-type elements so they are two different characteristic length scales (Fig. 3f).

\section{Velocity profiles}

\section{Velocity profiles measured at different positions}

To compare the velocity profiles measured at different positions in a repeating unit of roughness element, Fig. 4 depicts the mean wind speed $\bar{u}$, streamwise fluctuating velocity $\overline{u^{\prime \prime} u^{\prime \prime}} 1 / 2$, vertical fluctuating velocity $\overline{w^{\prime \prime} w^{\prime \prime}} 1 / 2$, and momentum flux $\overline{u^{\prime \prime} w^{\prime \prime}} 1 / 2$ over rib-type arrays of $\mathrm{AR}=1 / 4$ and cube-type arrays of $h: 4 l$. The velocities are normalized by the free-stream wind speed $U_{\infty}$. The wallnormal distance is measured from the roof-level $z-h$ which is then normalized by the TBL thickness $\delta$. The gradients of mean wind speed are similar over different measured positions. It is about $40 \%$ of $U_{\infty}$ at the roughness-element height $(z=h)$. The differences of the mean wind speed among individual profiles and their spatial average are less than $6 \%$. However, scattered data (deviations within $12 \%$ from the spatially averaged profiles) are found for ${\overline{u^{\prime \prime}}}^{\prime \prime} u^{1 / 2}$ and ${\overline{w^{\prime \prime} w^{\prime \prime}}}^{1 / 2}$ in the near-wall region $(z-h<0.1 \delta)$. It hence, demonstrates the inhomogeneous flows due to the presence of roughness elements in the lower TBL. This feature is in fact more noticeable for the vertical profiles of ${\overline{u^{\prime \prime} w^{\prime \prime}}}^{1 / 2}$. It highly varies (up to $80 \%$ deviation from the spatially average profiles) in the nearwall region, suggesting that significant dynamic effects are induced by individual roughness elements. The inhomogeneous flows are mainly located in $z-h<0.1 \delta$ over rib-type arrays and even lower in $z-h<0.05 \delta$ over cube-type arrays. A constant turbulent momentum flux region, which is defined as inertial sublayer (ISL), is revealed in $0.1 \delta<z-h<0.3 \delta$ over rib-type arrays and $0.05 \delta<z-h<0.15 \delta$ over cube-type arrays.

\section{Velocity profiles measured over different rough surfaces}

To compare the effect of rough-surface configurations on the dynamics, the spatially average profiles of mean wind speed $\langle\bar{u}\rangle$ (Fig. 5a), streamwise fluctuating velocity $\left\langle\overline{u^{\prime \prime} u^{\prime \prime}}\right\rangle^{1 / 2}$ (Fig. 5b), vertical fluctuating velocity $\left\langle\overline{w^{\prime \prime} w^{\prime \prime}}\right\rangle^{1 / 2}$ (Fig. 5c) and momentum flux $\left\langle\overline{u^{\prime \prime} w^{\prime \prime}}\right\rangle^{1 / 2}$ (Fig. 5d) over an entire repeating unit of roughness element. The mean wind speed profiles are generally similar over different surface configurations, nevertheless, noticeable differences are found in the lower TBL. The roof-level mean wind speeds over all the roughness elements are in the range of $0.35 U_{\infty}<\langle\bar{u}\rangle_{z=h}<0.5 U_{\infty}$.
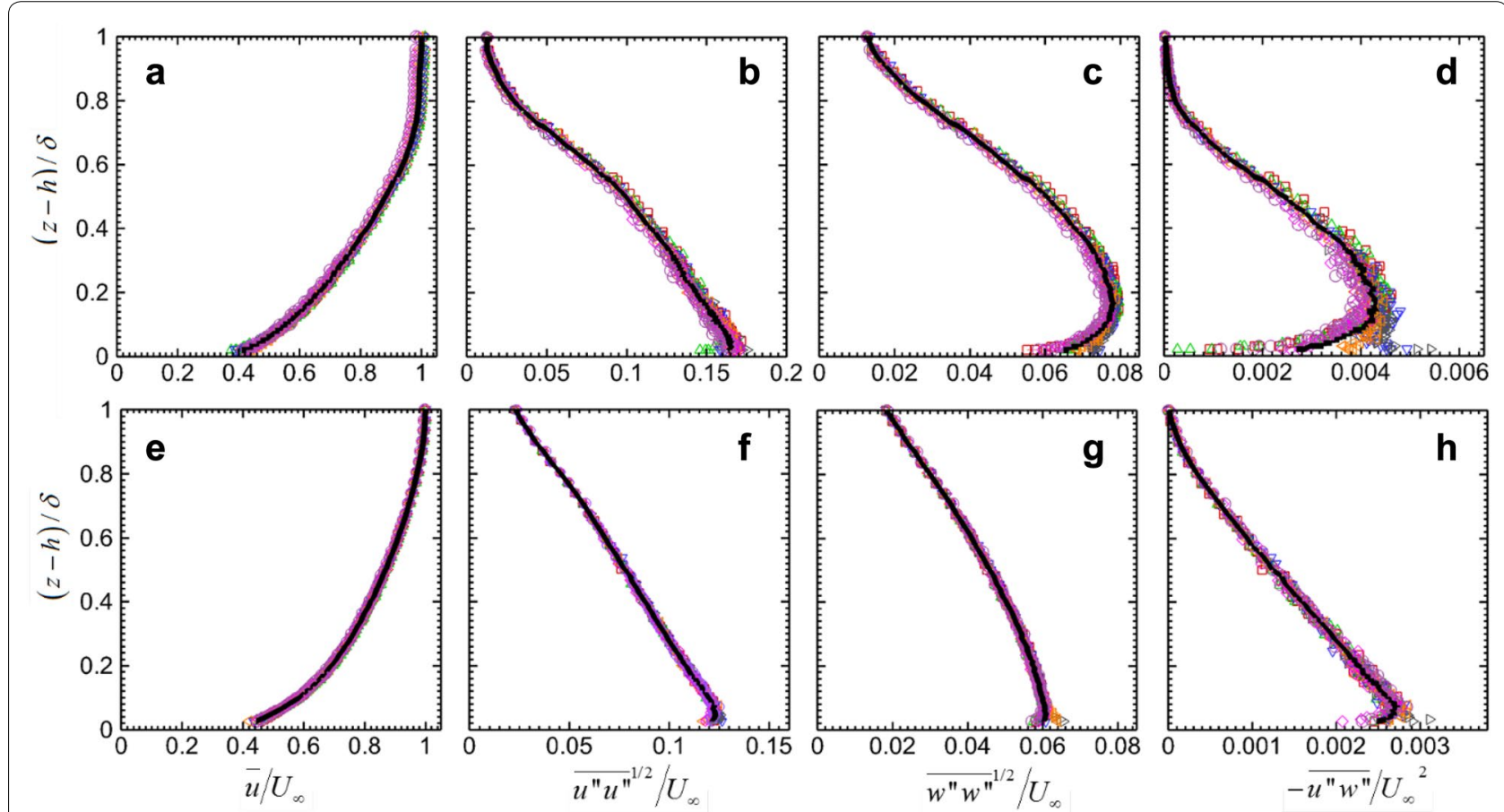

Fig. 4 Dimensionless vertical profiles of mean velocity $\bar{u}$, streamwise fluctuating velocity ${\overline{u^{\prime \prime}}}^{\prime \prime \prime}{ }^{1 / 2}$, vertical fluctuating velocity ${\overline{W^{\prime \prime}}}^{\prime \prime} w^{1 / 2}$ and momentum flux $\overline{u^{\prime \prime} W^{\prime \prime}}$ measured at different locations over the rib-type array $A R=1 / 4(\mathbf{a}-\mathbf{d})$ and cube-type array h:4l (e-h). P1 ( $\left.\square\right)$; P2 ( $\triangle$ ); P3 $(\nabla) ;$ P4 $(\triangleright) ;$ P5 $(\triangleleft) ;$ P6 $(\diamond) ; P 7(\bigcirc)$. Dark solid line is spatially average profile 

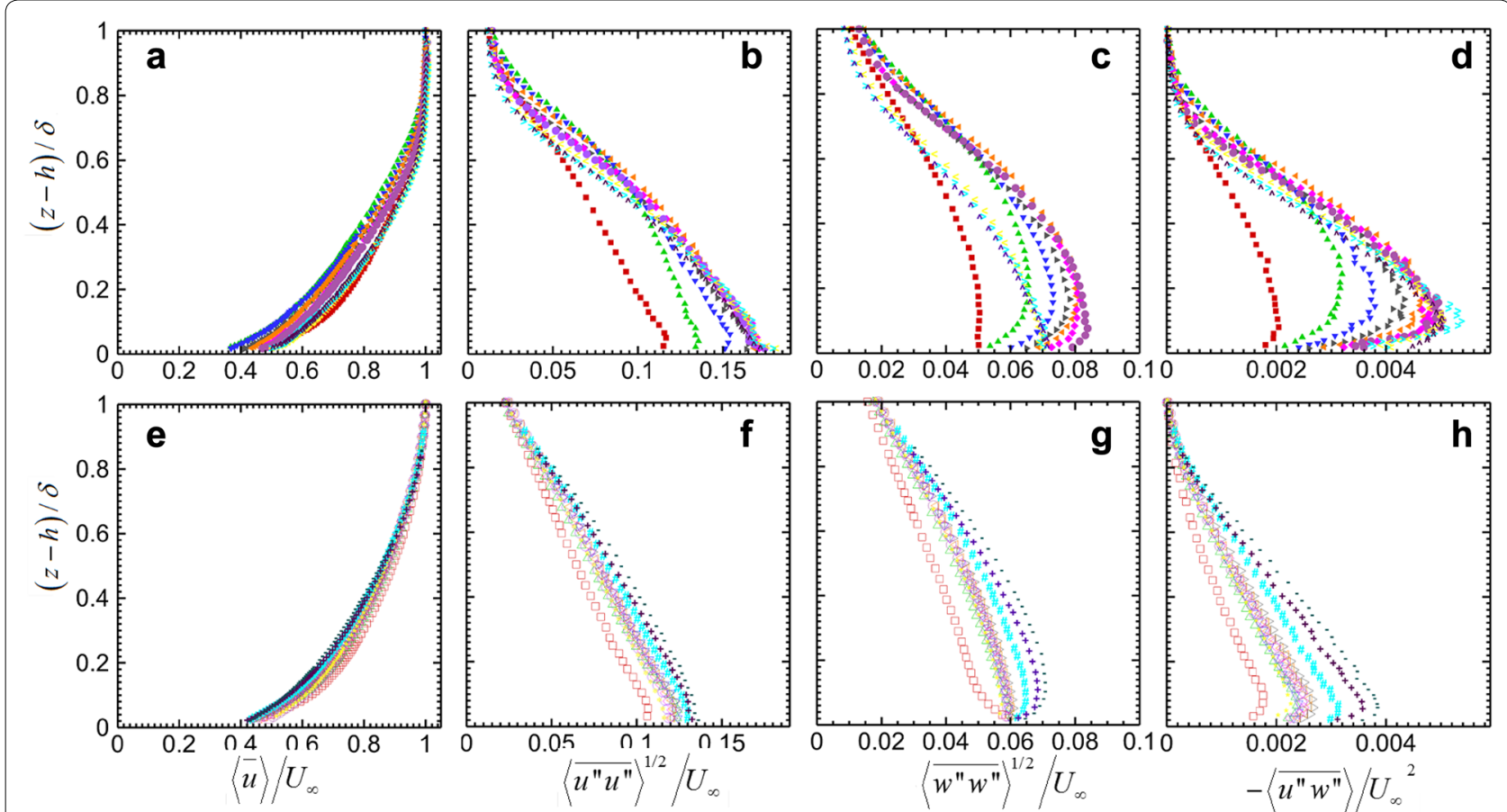

Fig. 5 Dimensionless spatio-temporally averaged vertical profiles of flow properties over rib-type and cube-type arrays expressed as functions of dimensionless wall-normal distance $(z-h) / \delta$. a, e Mean wind speed $\langle\bar{u}\rangle, \mathbf{b}, \mathbf{f}$ streamwise fluctuating velocity $\left\langle\overline{u^{\prime \prime} u^{\prime \prime}}\right\rangle^{1 / 2} ; \mathbf{c}$, g vertical fluctuating

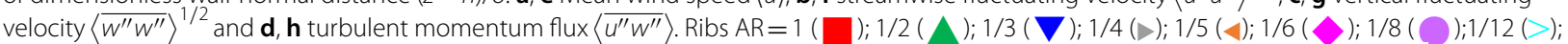
and 1/15 (^). Cubes $h: 1(\square) ; h: 2 l(\triangle) ; h: 3 /(\nabla) ; h: 4 l(\triangleright) ; h: 5 /(\triangleleft) ; h: 6 l(\diamond) ; h: 7 l(\bigcirc) ; h: 9 l(*) ; h: 4 l-D(\#) h: 4 l-T(+) ;$ and $h: 4 l-Q(-)$

There are notable variations of $\left\langle\overline{u^{\prime \prime} u^{\prime \prime}}\right\rangle^{1 / 2},\left\langle\overline{w^{\prime \prime} w^{\prime \prime}}\right\rangle^{1 / 2}$, and $\left\langle\overline{u^{\prime \prime} w^{\prime \prime}}\right\rangle^{1 / 2}$ over rib- and cube-type arrays, implying that the elevated roof-level turbulence intensity is attributed to the shear close to solid boundary. The variation in velocities over different rib- and cube-type arrays vanishes with increasing wall-normal distance. The mean wind speed profiles collapse in the outer TBL $(z-h>0.6 \delta)$ where the flows are barely affected by the surface roughness. The maxima of $\left\langle\overline{u^{\prime \prime} u^{\prime \prime}}\right\rangle^{1 / 2}$, $\left\langle\overline{w^{\prime \prime} w^{\prime \prime}}\right\rangle^{1 / 2}$ and $\left\langle\overline{u^{\prime \prime} w^{\prime \prime}}\right\rangle^{1 / 2}$ reside in the near-wall region $(z-h>0.1 \delta)$ that increase with widening roughnesselement separation. They reach a plateau (over the ribtype array of $\mathrm{AR}=1 / 8$ and the cube-type array of $h: 4 l$ ), then decrease thereafter with increasing the separation. It is because in the closely packed configurations (small separation among roughness elements), namely, skimming flow regime (Oke 1988), the flows seldom entrain into the cavity, resulting in a lower turbulence level. With increasing roughness-element separation, the turbulence level is enhanced by the interaction between the prevailing flows and cavity flows. However, with further increasing separation, the surface becomes smoother again as the roughness elements are sparsely distributed, which results in lower turbulence level. The high turbulence indicated strong shear over the top of roughness element.

\section{Quadrant analyses}

At the roof level, a substantial variation of turbulence level is observed over different sampling positions (Fig. 4) and over different rough-surface configurations (Fig. 5). To elucidate the momentum transfer between the prevailing flows and cavity flows, quadrant analyses are performed for data at the roof-level sampling points $(z=h)$. Based on the instantaneously measured components of fluctuating streamwise $u^{\prime \prime}$ and vertical $w^{\prime \prime}$ velocity, events of momentum flux transport are categorized into four quadrants, namely, outward interaction Q1 $\left(u^{\prime \prime}>0\right.$ and $\left.w^{\prime \prime}>0\right)$, ejection Q2 $\left(u^{\prime \prime}<0\right.$ and $\left.w^{\prime \prime}>0\right)$, inward interaction Q3 $\left(u^{\prime \prime}<0\right.$ and $\left.w^{\prime \prime}<0\right)$ and sweep Q4 $\left(u^{\prime \prime}>0\right.$ and $\left.w^{\prime \prime}<0\right)$ (Wallace et al. 1972; Lu and Willmarth 1973; Wallace 2016). The momentum flux can be calculated by:

$$
\overline{u^{\prime \prime} w^{\prime \prime}}=\int_{-\infty}^{+\infty} u^{\prime \prime} w^{\prime \prime} P\left(u^{\prime \prime}, w^{\prime \prime}\right) d u^{\prime \prime} d w^{\prime \prime},
$$




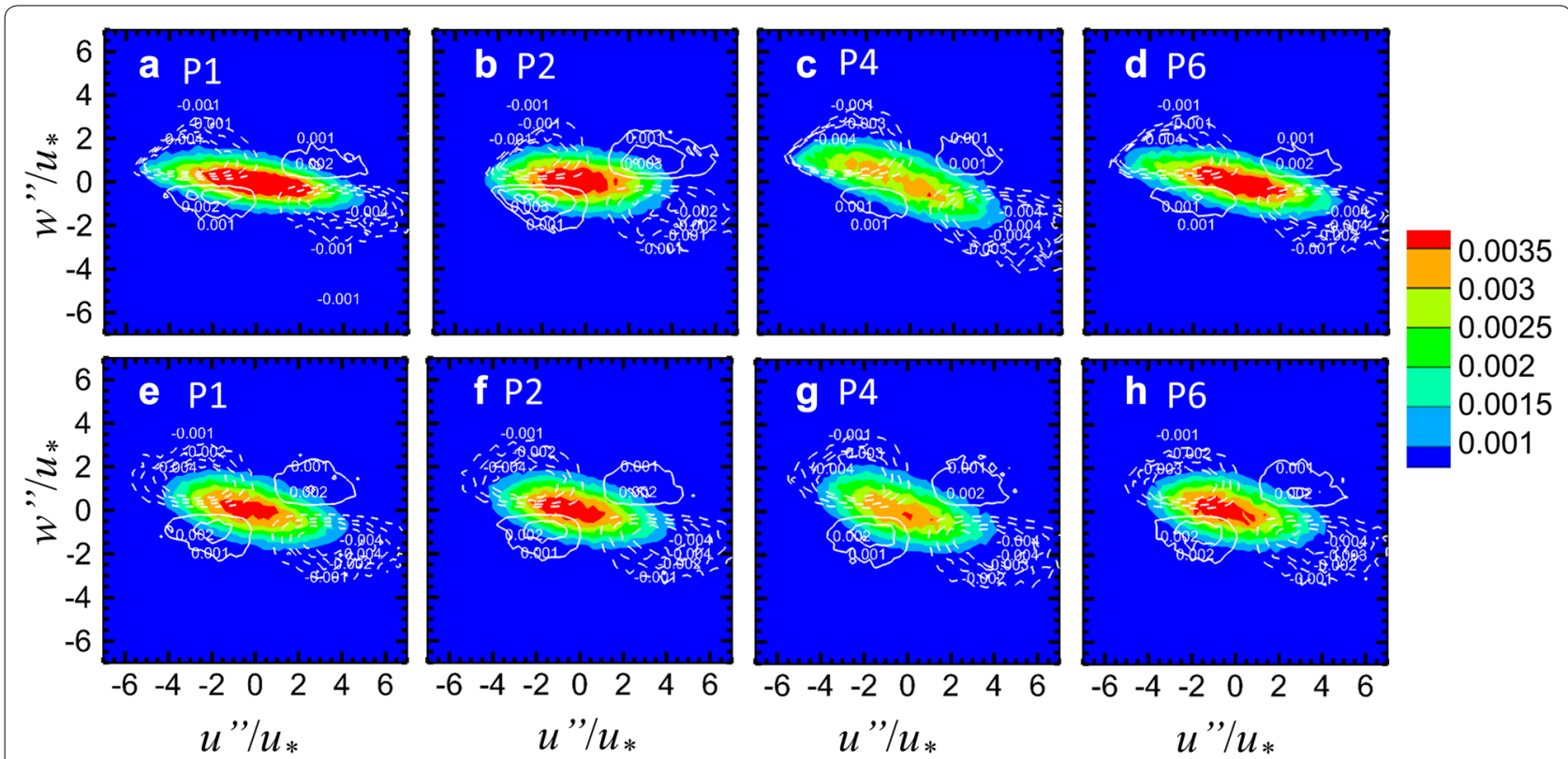

Fig. 6 Shaded contours of joint probability density function (JPDF) $P\left(u^{\prime \prime}, w^{\prime \prime}\right)$ and line contours of covariance integrand $u^{\prime \prime} w^{\prime \prime} P\left(u^{\prime \prime}, w^{\prime \prime}\right)$ at canopy level $(z=h)$ measured at different location over the rib-type array of $A R=1 / 4(\mathbf{a}-\mathbf{d})$ and cube-type array of $h: 4 /(\mathbf{e}-\mathbf{h})$

where $P\left(u^{\prime \prime}, w^{\prime \prime}\right)$ is the joint probability density function (JPDF) of the fluctuating velocity components $u^{\prime \prime}$ and $w^{\prime \prime}$; here $u^{\prime \prime} w^{\prime \prime} P\left(u^{\prime \prime}, w^{\prime \prime}\right)$ is the covariance integrand. The JPDF depicts the occurrence frequency of fluctuating velocities $u^{\prime \prime}$ and $w^{\prime \prime}$ in each quadrant event. The covariance integrand, on the other hand, illustrates the contribution of the total momentum flux to each quadrant. Figure 6 shows the roof-level JPDF and covariance integrand (Fig. 6a over rib-type arrays and Fig. 6e over cube-type arrays), leeward side (Fig. 6b, f), cavity top (Fig. 6c, g), and windward side (Fig. 6d, h). The JPDF is peaked at small fluctuating velocities over roughness elements and on the leeward/windward sides. It spreads out to Q2 and Q4 at cavity top. At the same time, the strength of Q2 and Q4 increases while Q1 and Q3 is suppressed (contour lines). The occurrence of Q2 and Q4 is more frequent than Q1 and Q3, indicating that the ejection Q2 $\left(u^{\prime \prime}<0\right.$ and $\left.w^{\prime \prime}>0\right)$ and sweep Q4 $\left(u^{\prime \prime}>0\right.$ and $\left.w^{\prime \prime}<0\right)$ dominate the mechanism of roof-level transport processes which is in line with previous studies (Wallace 2016). The larger values of covariance integrand Q2 and Q4 at the cavity top suggest that aged air removal $\left(w^{\prime \prime} \geq 0\right)$ and fresh air entrainment $\left(w^{\prime \prime} \leq 0\right)$ are driven by decelerating $\left(u^{\prime \prime} \leq 0\right)$ and accelerating $\left(u^{\prime \prime} \geq 0\right)$ air masses, respectively.

Figure 7 compares the JDPF and covariance integrand at the cavity top (P4 in Fig. 2) over different rough-surface configurations. It is clearly shown that the JDPF spreads out in the directions of ejection Q2 and sweep Q4 with increasing drag coefficient while Q1 and Q3 are suppressed accordingly. The covariance integrands of Q2 and Q4 are strengthened with increasing aerodynamic resistance. It is thus suggested that the air entrainment and removal are enhanced over rougher surfaces, resulting in more efficient roof-level ventilation.

\section{Frequency spectra}

Frequency spectra are calculated to examine the rooflevel turbulence motion scales (El-Gabry 2014). Similar to the quadrant analyses using the data at cavity top (P4 in Fig. 2), the instantaneous flow signal is proceeded using Fast Fourier Transform (FFT) to convert it from the time domain to the frequency domain (Storey 2002; ElGabry 2014). As shown in Fig. 8, the energy spectra of $u^{\prime \prime}$ are higher than those of $w^{\prime \prime}$ over an order of magnitude for $f \times h / u_{*}<1$, but they decrease sharply when $f \times h / u_{*}>1$ for the streamwise fluctuating velocity $u^{\prime \prime}$ and $f \times h / u_{*}>10$ for the vertical fluctuating velocity $w^{\prime \prime}$. The inertial subrange is also clearly depicted for both $u^{\prime \prime}$ and $w^{\prime \prime}$, showing the energy cascade in different scales of motions in isothermal conditions. The spectra of $u^{\prime \prime}$ and $w^{\prime \prime}$ are comparable for $f \times h / u_{*}>10$ because of the isotropic small-scale motions. Comparing the energy spectra over different sampling positions (Fig. 8a, b for rib-type arrays and Fig. 8e, $\mathrm{f}$ for cube-type arrays), energy spectra are higher for $u^{\prime \prime}$ and $w^{\prime \prime}$ at the cavity top (P4) than those at roof level (P1). Large-scale motions enhance the turbulent transport at the cavity top. Comparing the energy spectra over different rough surfaces, large-scale 

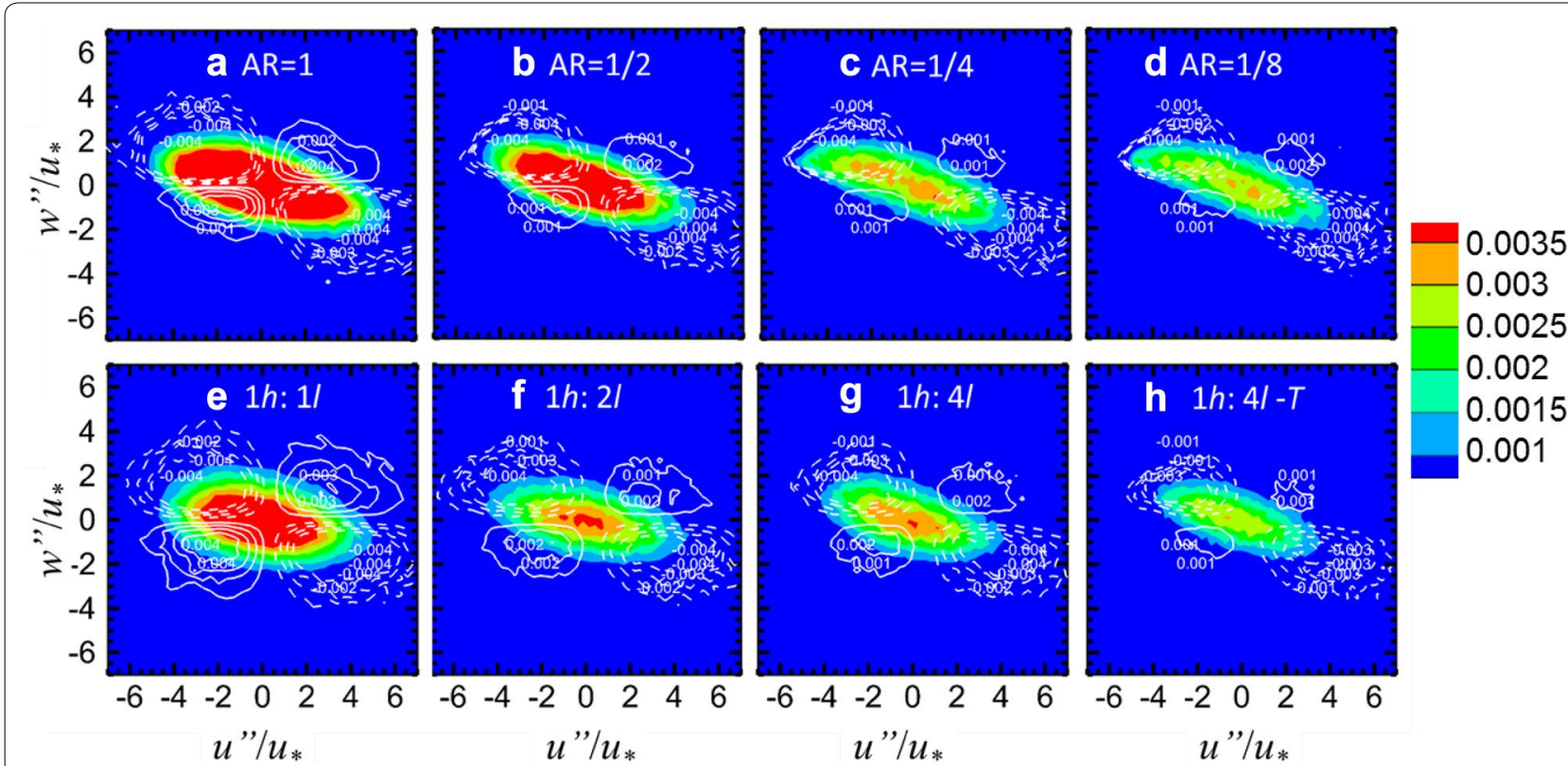

0.002

0.0015

0.001

Fig. 7 Shaded contours of joint probability density function (JPDF) $P\left(u^{\prime \prime}, w^{\prime \prime}\right)$ and line contours of covariance integrand $u^{\prime \prime} w^{\prime \prime} P\left(u^{\prime \prime}, w^{\prime \prime}\right)$ at canopy height $(z=h)$ of $\mathrm{P} 4$ measured over the rib-type arrays of $\mathbf{a} A R=1, \mathbf{b} A R=1 / 2, \mathbf{c} A R=1 / 4$, and $\mathbf{d} A R=1 / 8$ and cube-type arrays of $\mathbf{e} 1 \mathrm{~h}: 11, \mathbf{f} 1 \mathrm{~h}: 2 \mathrm{l}, \mathbf{g}$ $1 \mathrm{~h}: 4 \mathrm{l}$, and $\mathbf{h} 1 \mathrm{~h}: 4 \mathrm{l}-\mathrm{T}$

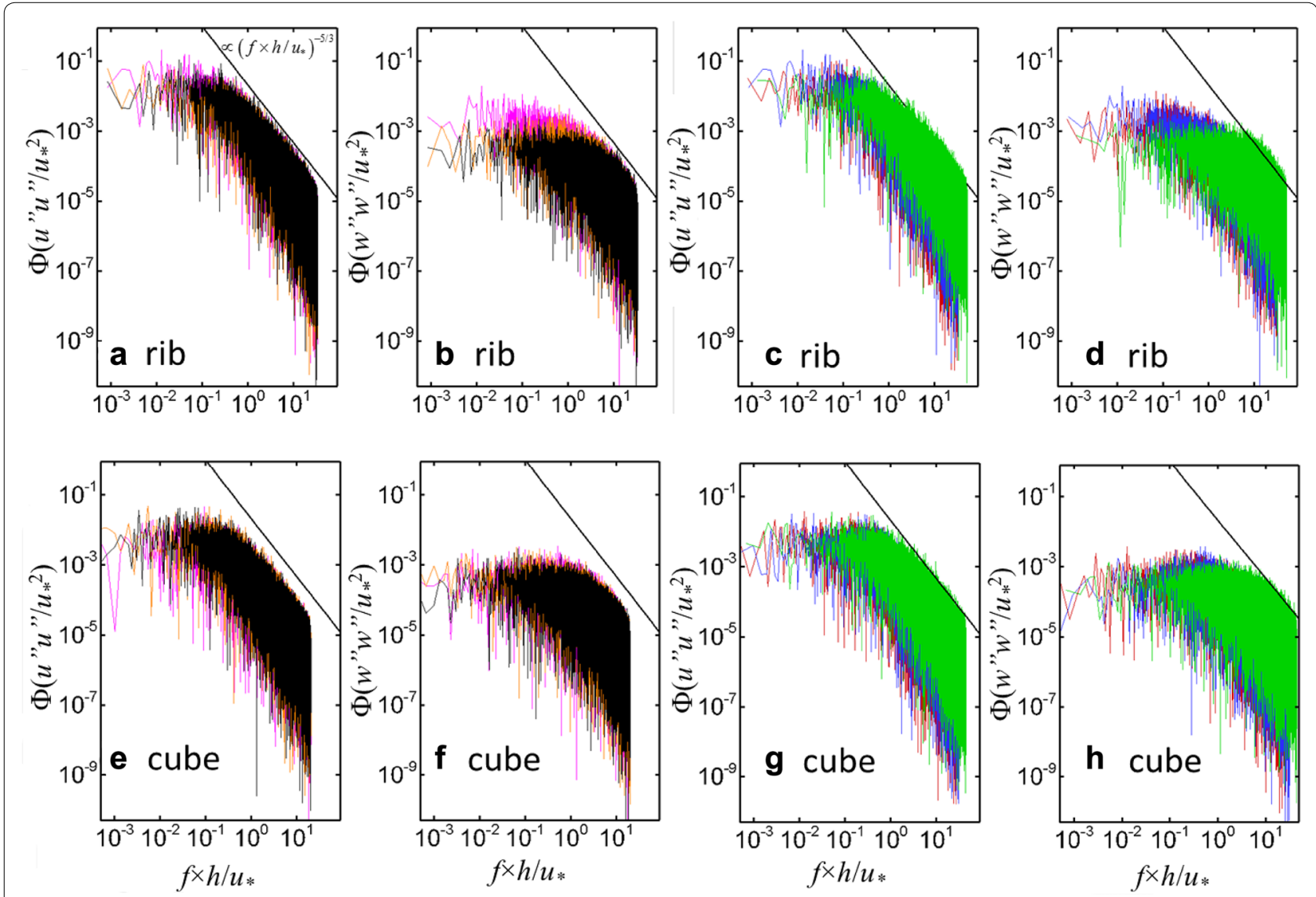

Fig. 8 Frequency spectra of dimensionless streamwise $\Phi\left(u^{\prime \prime} u^{\prime \prime} / u_{*}^{2}\right)$ and vertical $\Phi\left(w^{\prime \prime} w^{\prime \prime} / u_{*}^{2}\right)$ turbulence intensities at canopy level $z=h$ over hypothetical urban areas. $\mathbf{a}, \mathbf{b}$ Are ribs of $A R=1 / 4$ and $(\mathbf{e}, \mathbf{f})$ are cubes for l:4h measured at P1 (black), P2 (orange), P4 (magenta). $\mathbf{c}, \mathbf{d}$ Are ribs for $A R=A R=1 / 2$ (green), $A R=1 / 4$ (blue), and $A R=1 / 8$ (red) measured at P4. g, h Are cubes for $h: 1 /$ (green), $h: 4 /$ (blue), and $h: 4 /-T$ (red) measured at P4 
turbulence is found with increasing drag coefficient over rib-type arrays, especially for vertical fluctuating velocity $w^{\prime \prime}$ (taking Case AR $=1, \mathrm{AR}=1 / 12$, and $h: l, h: 4 l-D$ for exampling). The feature is mild for cube-type arrays, probably because the drag coefficient is similar among different cubical roughness elements. These results suggest that the vertical transports are governed by larger scale turbulence with increasing drag coefficient in which the momentum transports are enhanced.

\section{Conclusions}

TBLs over rib- and cube-type arrays are developed in the wind tunnel to examine the flow and turbulence characteristics. For the aerodynamic parameters, a notable trend is observed that roughness length $z_{0}$ increases with increasing drag coefficient $C_{d}$ while displacement height $d$ varies significantly with increasing $C_{d}$. Significant variations of fluctuating velocities and momentum flux are found in the near-wall region, demonstrating the inhomogeneous flows due to the presence of roughness elements in the bottom of TBL. Comparing the velocities over different rough surfaces, it is found that the spatially averaged fluctuating streamwise velocity $\left\langle\overline{u^{\prime \prime} w^{\prime \prime}}\right\rangle^{1 / 2}$, fluctuating vertical velocity $\left\langle\overline{w^{\prime \prime} w^{\prime \prime}}\right\rangle^{1 / 2}$ and momentum flux $\left\langle\overline{u^{\prime \prime} w^{\prime \prime}}\right\rangle$ in the near-wall region increase with widening separation among roughness elements, reach a plateau (over rib-type array of $\mathrm{AR}=1 / 8$ and cube-type array of $h: 4 l)$, then finally decrease with further increasing separation between roughness elements. Quadrant analyses and frequency spectra show that the flow entrainment and air removal are enhanced over rougher surfaces. Larger scale motions of turbulence also effectuate roof-level ventilation over urban areas.

\section{Author contributions}

ZM performed the experiments and drafted the manuscript. CHL performed data interpretation and drafted the manuscript. Both authors read and approved the final manuscript.

\section{Acknowledgements}

Not applicable.

\section{Competing interests}

The authors declare that they have no competing interests.

\section{Availability of data and materials}

The data are available from the corresponding author on reasonable request.

Ethics approval and consent to participate

Not applicable.

\section{Funding}

This study is partly supported by the General Research Fund (GRF) 17210115 of The Hong Kong Research Grants Council (RGC).

\section{Publisher's Note}

Springer Nature remains neutral with regard to jurisdictional claims in published maps and institutional affiliations.
Received: 27 February 2018 Accepted: 18 June 2018

Published online: 26 June 2018

\section{References}

Britter RE, Hanna SR (2003) Flow and dispersion in urban areas. Annu Rev Fluid Mech 35:469-496

Bruun H (1971) Interpretation of a hot wire signal using a universal calibration law. J Phys E Sci Instrum 4:225

Castro IP, Cheng H, Reynolds R (2006) Turbulence over urban-type roughness: deductions from wind-tunnel measurements. Bound Layer Meteorol 118:109-131

Cermak JE (1981) Wind tunnel design for physical modelling of atmospheric boundary layers. J Eng Mech Div ASCE 108:523-642

Cheng H, Castro IP (2002a) Near wall flow over urban-like roughness. Bound Layer Meteorol 104:229-259

Cheng H, Castro IP (2002b) Near-wall flow development after a step change in surface roughness. Bound Layer Meteorol 105:411-432

Cheng H, Hayden P, Robins AG, Castro IP (2007) Flow over cube arrays of different packing densities. JWind Eng Ind Aerodyn 95:715-740

El-Gabry LA, Thurman DR, Poinsatte PE (2014) Procedure for determining turbulence length scales using hotwire anemometry. Technical Report NASAV TM-2014-218403, NASA Glenn Research Center, Cleveland, OH, United States

Ho YK, Liu CH (2017) A wind tunnel study of flows over idealised urban surfaces with roughness sublayer corrections. Theor Appl Climatol 130(1-2):305-320

Jiménez J (2004) Turbulent flows over rough walls. Annu Rev Fluid Mech 36:173-196

Kozmar H (2010) Scale effects in wind tunnel modelling of an urban atmospheric boundary layer. Theor Appl Climatol 100:153-162

Krogstad PA, Antonia R, Browne L (1992) Comparison between rough- and smooth-wall turbulent boundary layers. J Fluid Mech 245:599-617

Liu CH, Ng T, Wong CCC (2015) A theory of ventilation estimate over hypothetical urban areas.J Hazard Mater 296:9-16

Lu S, Willmarth W (1973) Measurements of the structure of the Reynolds stress in a turbulent boundary layer. J Fluid Mech 60(3):481-511

Manes C, Poggi D, Ridolfi L (2011) Turbulent boundary layers over permeable walls: scaling and near-wall structure. J Fluid Mech 687:141-170

Mo Z, Liu CH (2018) Wind tunnel measurements of pollutant plume dispersion over hypothetical urban areas. Build Environ. 132:357-366

NI (2018) National Instrument National Instruments, Austin, TX http://www. ni.com

Oke TR (1988) Street design and urban canopy layer climate. Energy Build 11:103-113

Perry AE, Lim KL, Henbest SM (1987) An experimental study of the turbulence structure in smooth- and rough-wall boundary layers. J Fluid Mech $177: 437-466$

Placidi M, Ganapathisubramani B (2015) Effects of frontal and plan solidities on aerodynamic parameters and the roughness sublayer in turbulent boundary layers. J Fluid Mech 782:541-566

Pope SB (2000) Turbulent flows. Cambridge University Press, Cambridge

Raupach MR, Antonia RA, Rajagopalan S (1991) Rough-wall turbulent boundary layers. Appl Mech Rev 44(1):1-25

Salizzoni P, Soulhac L, Mejean P, Perkins RJ (2008) Influence of a two-scale surface roughness on a neutral turbulent boundary layer. Bound Layer Meteorol 127:97-110

Storey BD (2002) Computing Fourier series and power spectrum with Matlab. TEX paper

Wallace JM (2016) Quadrant analysis in turbulence research: history and evolution. Annu Rev Fluid Mech 48:131-158

Wallace JM, Brodkey RS, Eckelmann H (1972) The wall region in turbulent shear flow. J Fluid Mech 54:39-48 